\title{
Prompt Photons in Photoproduction
}

\author{
Krzysztof Nowak and Katharina Müller \\ University of Zürich - Physics Department \\ Winterthurerstrasse 190, CH - 8057 Zürich - Switzerland
}

\begin{abstract}
The yield of prompt photons has been measured in the photoproduction regime of electron-proton scattering at HERA. The analysis is based on data taken by the H1 experiment with a total integrated luminosity of $340 \mathrm{pb}^{-1}$. Cross sections have been measured for photons with transverse momentum and pseudorapidity in the range $6<E_{T}^{\gamma}<15 \mathrm{GeV}$ and $-1.0<\eta^{\gamma}<2.4$, respectively. Cross sections for events with an additional hadronic jet are measured as a function of $E_{T}^{j e t}, \eta^{j e t}, x_{\gamma}$ and $x_{p}$. Additionally, the transverse correlation between the photon and the jet is studied. The results are compared with predictions of a next-to-leading order calculation and a calculation based on $k_{T}$ factorisation approach.
\end{abstract}

\section{Introduction}

Isolated photons emerging from the hard subprocess $e p \rightarrow e \gamma X$, so called prompt photons, are a powerful probe of the underlying dynamics. They generally require smaller corrections for hadronisation than measurements relying on jets.

The prompt photon production has been studied at HERA by both the H1 $[2,3]$ and the ZEUS $[4,5,6]$ collaborations. In photoproduction, both experiments found that the inclusive prompt photon production is underestimated by next-to-leading order (NLO) QCD calculations, while there is a reasonable agreement with results for photons associated with jets. In deep inelastic scattering (DIS), a leading order QCD calculation significantly underestimates the production of isolated photons and of photons associated with jets. NLO predictions are only available for the latter and also underestimate the cross section.

New results are presented on prompt photons in photoproduction. The data used for the measurement have been collected by the H1 detector in the years $2004-2007$ with a total luminosity of $340 \mathrm{pb}^{-1}$. Compared to previous $\mathrm{H} 1$ measurement statistics is increased by a factor of three. In addition the phase space is extended to larger pseudorapidities of the photon and to lower event inelasticities.

\section{Event selection}

The analysis is based on photoproduction events with the electron scattered under small angle, such that it is not detected in the main experimental apparatus. Events are triggered by a photon candidate, being a highly energetic, electromagnetic cluster in the liquid argon (LAr) calorimeter [7].

Low $Q^{2}$ DIS events are removed by excluding events with an electron in the backward calorimeter, high $Q^{2}$ events are suppressed by removing events with an electron candidate in the LAr calorimeter not previously identified as photon candidate. The DIS background is below $1.5 \%$ in the final sample.

The inelasticity is restricted to $0.1<y_{h}=\Sigma\left(E-p_{z}\right) / 2 E_{e}<0.7$ where $E_{e}$ is the energy of the incoming electron and the sum runs over the energy $(E)$ and the longitudinal momentum 
$\left(p_{z}\right)$ of all detected final state particles. In order to correctly determine the vertex position at least two well reconstructed central tracks are required.

Photon candidates are defined as electromagnetic clusters in the LAr calorimeter with transverse energy $6<E_{T}^{\gamma}<15 \mathrm{GeV}$ and pseudorapidity ${ }^{\mathrm{a}}-1.0<\eta^{\gamma}<2.4$. Charged particles are removed by a combined condition based on the information from the central tracking detector and the central inner proportional chamber [8]. The cluster has to be compact with a transverse radius of less than $6 \mathrm{~cm}$.

The main experimental difficulty is the separation of photons from neutral mesons, mainly $\pi^{0}$ or $\eta$, decaying into multi-photon final states. The background contamination is greatly reduced by the isolation requirement, that the photon carries more than $90 \%$ of the transverse energy of the jet in which it is contained, i.e. $z=E_{T}^{\gamma} / E_{T}^{\text {photon-jet }}>0.9$. A cut on the invariant mass of two neighbouring clusters is applied which removes photons from $\pi^{0}$ decays reconstructed in separate clusters as well as converted photons.

For the exclusive sample, events are selected with a photon candidate accompanied by at least one hadronic jet with $E_{T}^{\text {jet }}>4.5 \mathrm{GeV}$ and $-1.3<\eta^{\text {jet }}<2.3$. Jets are found using the inclusive $k_{T}$ algorithm [9] with the separation parameter $R_{0}=1$. Additional observables $x_{\gamma}^{L O}, x_{p}^{L O}, p_{\perp}$ and $\Delta \Phi$ are defined for the exclusive sample which give more insight into the underlying partonic process.

The estimators $x_{\gamma}^{L O}$ and $x_{p}^{L O}$, which in the LO approximation correspond to the longitudinal momentum fractions of the partons in the photon and the proton, respectively, are defined as

$$
x_{\gamma}^{L O}=E_{T}^{\gamma}\left(e^{-\eta^{j e t}}+e^{-\eta^{\gamma}}\right) /\left(2 y_{h} E_{e}\right)
$$

and

$$
x_{p}^{L O}=E_{T}^{\gamma}\left(e^{\eta^{j e t}}+e^{\eta^{\gamma}}\right) /\left(2 E_{p}\right) .
$$

The above definitions make use of the energy of the photon which has a better resolution than the energy of the jet and reduce infrared sensitivity for $x_{\gamma} \rightarrow 1$. It should be noted that $x_{\gamma}^{L O}$ may become larger than one.

\begin{tabular}{|c|c|}
\hline H1 Prompt Photon Phase Space \\
\hline Inclusive & Exclusive \\
\hline $6<E_{T}^{\gamma}<15 \mathrm{GeV}$ \\
$-1.0<\eta^{\gamma}<2.4$ \\
$z=E_{T}^{\gamma} / E_{T}^{j e t}>0.9$ \\
$Q^{2}<1 \mathrm{GeV}^{2}$ \\
$0.1<y<0.7$ \\
$-\quad \mid \quad E_{T}^{\text {jet }}>4.5 \mathrm{GeV}$ \\
$-\quad$ & $-1.3<\eta^{j e t}<2.3$ \\
\hline
\end{tabular}

Table 1: Phase space for the measurement of inclusive and exclusive isolated prompt photon cross sections. Kinematics are defined in the H1 laboratory frame.
The two observables $p_{\perp}$ and $\Delta \Phi$ describe the transverse correlation between the photon and the jet. $\Delta \Phi$ is the azimuthal acoplanarity between the photon and the jet, and $p_{\perp}$ is the photon momentum perpendicular to the jet direction in the transverse plane

$$
p_{\perp} \equiv \frac{\left|\vec{p}_{T}^{\gamma} \times \vec{p}_{T}^{j e t}\right|}{\left|\vec{p}_{T}^{j e t}\right|}=E_{T}^{\gamma} \cdot \sin \Delta \Phi
$$

At leading order the prompt photon and the jet are back-to-back and $p_{\perp}$ equals zero for direct processes. $\Delta \Phi$ is strongly correlated with $p_{\perp}$ but is less sensitive to the energies of the photon and the jet.

\footnotetext{
aThe pseudorapidity $\eta$ being defined as $\eta=-\ln \tan (\theta / 2)$ with $\theta$ being the polar angle in the H1 coordinate system with the origin in the nominal ep interaction point and direction of the proton beam defining the positive $z$-axis.
} 


\section{Photon signal extraction}

Background to the analysis of prompt photons mainly arises from energetic photons emitted in a decay of hadrons like $\pi^{0}$ and $\eta$. The photon signal is extracted from the sample with photon candidates by a multivariate analysis (MVA) using the shower shape variables as input. It is based on the fact that electromagnetic showers in the calorimeter initiated by isolated photons typically are more narrow, symmetric and start off slightly deeper in the calorimeter than background showers. Six different shower shape variables are combined in the MVA producing discriminator distributions. They depend on the energy $E_{T}^{\gamma}$ and pseudorapidity $\eta^{\gamma}$ of the photon candidate.

Isolated photon cross sections are extracted using a regularised unfolding procedure $[10,11,12]$. The migration matrix is determined with the help of prompt photon Monte Carlo events generated with PYTHIA 6.2 [13] and passed through a GEANT [14] simulation of the $\mathrm{H} 1$ detector.

\section{Results}

Table 1 summarises the inclusive and exclusive phase space for the prompt photon cross sections presented below.

Bin averaged differential cross sections are presented in figures 1 to 4 . The inner error bars of the measured cross section correspond to the uncorrelated errors, including the statistical error. The outer error bars include the correlated errors added in quadrature. For all measurements the total uncertainty is dominated by the systematic error coming from the uncertainty of the description of the shower shape variables in the simulation.

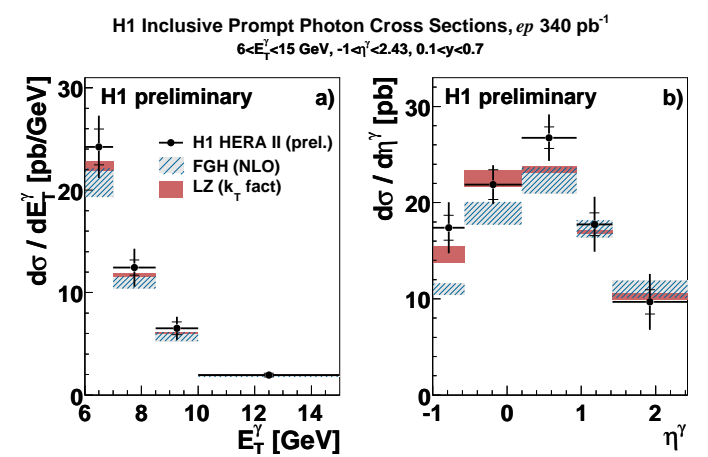

Figure 1: Inclusive differential prompt photon cross sections $d \sigma / d E_{T}^{\gamma}$ (a) and $d \sigma / d \eta^{\gamma}$ (b) in the inclusive phase space specified in table 1 . The data is compared to a NLO pQCD calculation and to a calculation based on the $k_{T}$ factorisation approach.
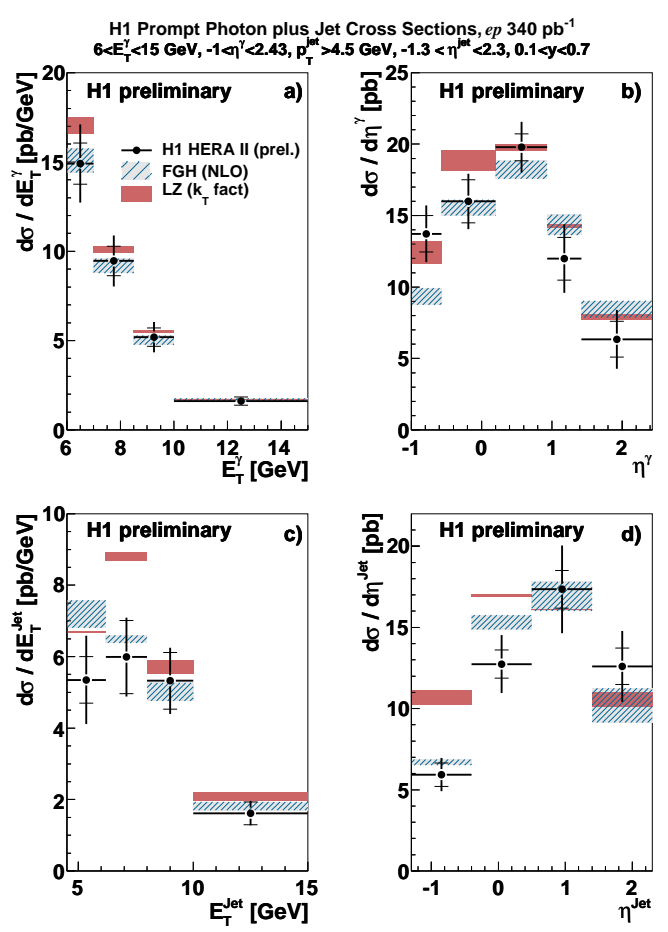

Figure 2: Exclusive differential prompt photon cross sections $d \sigma / d E_{T}^{\gamma}$ (a), $d \sigma / d \eta^{\gamma}$ (b), $d \sigma / d E_{T}^{j e t}(\mathrm{c})$ and $d \sigma / d \eta^{j e t}(\mathrm{~d})$ in the exclusive phase space specified in table 1 . 
All the results are compared to two sets of calculations, both corrected for hadronisation and multiple interaction effects: a next-to-leading order calculation by Fontannaz-Guillet-Heinrich (FGH) $[15,16]$ and a calculation based on the $k_{T}$-factorisation approach by Zotov-Lipatov (LZ) [17].

Differential inclusive cross sections $d \sigma / d E_{T}^{\gamma}$ and $d \sigma / d \eta^{\gamma}$ are presented in Fig. 1. The LZ calculation gives a nice description of the data, whereas the FGH calculation is below the data for backward photons $\left(\eta^{\gamma}<0.2\right)$. The PYTHIA prediction is significantly below the measurement, but not presented in the figures.

Cross sections for the production of an isolated prompt photon together with a hadronic jet are presented in Fig. 2 as a function of the variables $E_{T}^{\gamma}, \eta^{\gamma}, E_{T}^{j e t}$ and $\eta^{j e t}$. Both calculations give a reasonable description of the $\eta^{\gamma}$ and $E_{T}^{\gamma}$ cross sections while only the NLO calculation describes well $E_{T}^{j e t}$ and $\eta^{j e t}$ of the associated hadronic jet. Here, the LZ prediction is too high for jets with $\eta^{\text {jet }}<0.5$.

Figure 3 shows the photon plus jet cross section as a function of the estimators $x_{\gamma}^{L O}$ and $x_{p}^{L O}$. Whereas the $x_{\gamma}^{L O}$ distribution is well described by both predictions, the LZ calculation is higher than the data at lowest $x_{p}^{L O}$.

Figure 4 shows the cross sections for two observables describing the transverse correlation between the photon and the jet, $p_{\perp}$ and $\Delta \Phi$. Both variables are particularly sensitive to higher order gluon emission. The sample is split into a sample with $x_{\gamma}^{L O}>0.8$ (Figs. $4 \mathrm{a}$ and $4 \mathrm{~b}$ ) where the direct interaction of a photon with the proton dominates and a sample with $x_{\gamma}^{L O}<0.8$ (Figs. 4c and $4 \mathrm{~d}$ ) with a significant contribution of events with a resolved photon.

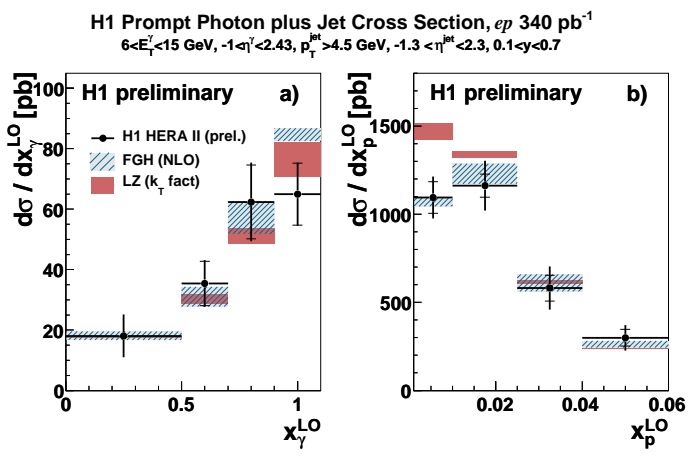

Figure 3: Exclusive differential prompt photon cross sections $d \sigma / d x_{\gamma}^{L O}$ (a) and $d \sigma / d x_{p}^{L O}$ (b) in the exclusive phase space specified in table 1.
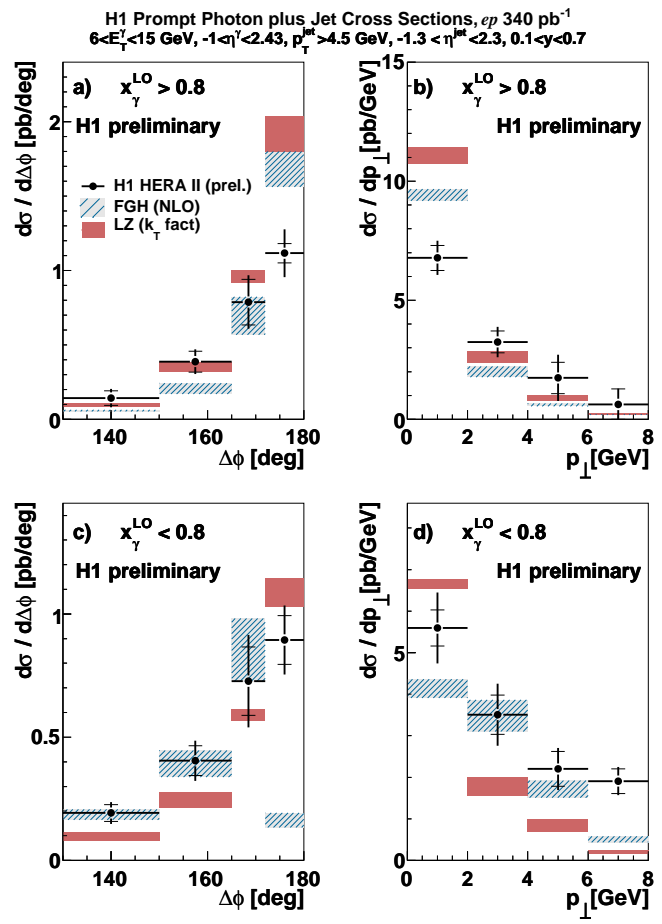

Figure 4: Exclusive differential prompt photon cross sections $d \sigma / d \Delta \Phi$ (a) and (c) and $d \sigma / d p_{\perp}$ (b) and $(d)$ in the exclusive phase space specified in table 1. (a) and (b) show cross section for $x_{\gamma}^{L O}>0.8$, while (c) and (d) for $x_{\gamma}^{L O}<0.8$. 
Both predictions overestimate the back-to-back configuration for $x_{\gamma}^{L O}>0.8$ but describe the tails reasonably well. For $x_{\gamma}^{L O}<0.8$, the $p_{\perp}$ distribution is slightly broader than for $x_{\gamma}^{L O}>0.8$, which reflects increased contributions from events with a resolved photon or from photons radiated from quarks in di-jet events. The FGH calculation gives a reasonable description of the distributions for $x_{\gamma}^{L O}<0.8$, except for the highest bins in $p_{\perp}$ and $\Delta \Phi$. The region $\Delta \Phi \rightarrow 180$ is sensitive to multiple soft gluon radiation which limits the validity of fixed order calculations [18]. The LZ calculation includes multiple soft gluon radiation in the initial state before the hard subprocess and describes $\Delta \Phi>170^{\circ}$ but predicts a significantly lower contribution of events in the tails of both distributions.

\section{Conclusions}

The photoproduction of prompt photons, both inclusively and associated with jets, is measured by the $\mathrm{H} 1$ collaboration using an integrated luminosity of $340 \mathrm{pb}^{-1}$. The data are compared to a NLO calculation and to a calculation based on the $k_{T}$ factorisation approach. For the inclusive sample the shapes of the $d \sigma / d E_{T}^{\gamma}, d \sigma / d \eta^{\gamma}$ distributions are described within errors, except for low $\eta^{\gamma}$ where the NLO prediction is significantly below the data. For the exclusive sample, differential cross sections are measured as a function of $E_{T}^{\gamma}, \eta^{\gamma}$, $\eta^{j e t}, E_{T}^{j e t}, x_{\gamma}^{L O}$ and $x_{p}^{L O}$. While the NLO calculation describes the measured shapes reasonably well, the calculation based on the $k_{T}$ factorisation approach significantly overestimates backward jets and low momentum fractions of the parton in the proton. Transverse correlations between the jet and the photon are investigated by measurements of $\Delta \Phi$ and $p_{\perp}$ dependent cross sections with deficits found in both calculations.

\section{References}

[1] Slides: http: //indico. cern. $\mathrm{ch} /$ contributionDisplay $\cdot$ py? contribId=283\&sess ionId=3\&conf $I d=53294$

[2] A. Aktas et al., Eur. Phys. J. C38 437 (2005) [hep-ex/0407018].

[3] F. D. Aaron et al., Eur. Phys. J. C54 371 (2008) [arXiv:0711.4578[hep-ex]].

[4] J. Breitweg et al., Phys. Lett. B 472 1, 175 (2000) [hep-ex/9910045].

[5] S. Chekanov et al., Phys. Lett. B 51119 (2001) [hep-ex/0104001].

[6] S. Chekanov et al., Eur. Phys. J. C49 511 (2007) [hep-ex/0608028].

[7] B. Andrieu et al., Nucl. Instrum. Meth. A336 460 (1993).

[8] J. Becker et al., Nucl. Instrum. Meth. A 586190 (2008) [arXiv:physics/0701002].

[9] S. D. Ellis, D. E. Soper, Phys. Rev. D 483160 (1993) [arXiv:hep-ph/9305266].

[10] D. L. Phillips, J. Assoc. Comput. Mach, 984 (1962).

[11] A. N. Tikhanov, Soviet Math. Dokl., 41035 (1963); English translation of Dokl. Akad. Nauk. SSSR, 151 (1963) 501.

[12] V. Blobel, An unfolding method for high energy physics experiments, Proc. Advanced Statistical Techniques in Particle Physics, Durham (2002).

[13] T. Sjöstrand et al., PYTHIA 6.2 Physics and Manual [hep-ph/0108264].

[14] GEANT 3, R.Brun et al., CERN_DD/EE/84-1.

[15] M. Fontannaz, J. P. Guillet and G. Heinrich, Eur. Phys. J. C 21303 (2001) [arXiv:hep-ph/0105121].

[16] M. Fontannaz and G. Heinrich, Eur. Phys. J. C 34, 191 (2004) [arXiv:hep-ph/0312009].

[17] A. V. Lipatov and N. P. Zotov, Phys. Rev. D 72054002 (2005) [arXiv:hep-ph/0506044].

[18] Gudrun Heinrich, private communication.

DIS 2009 